\title{
Negative pressure wound therapy enhances bone regeneration compared with conventional therapy in a rabbit radius gap-healing model
}

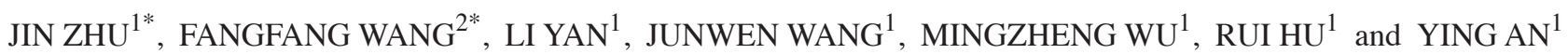 \\ ${ }^{1}$ Department of Orthopedics, Wuhan Fourth Hospital, Puai Hospital, Tongji Medical College, \\ Huazhong University of Science and Technology; ${ }^{2}$ Department of Ophthalmology, Tongji Hospital, \\ Tongji Medical College, Huazhong University of Science and Technology, Wuhan, Hubei 430000, P.R. China
}

Received September 4, 2019; Accepted October 14, 2020

DOI: 10.3892/etm.2021.9905

\begin{abstract}
Negative pressure wound therapy (NPWT) has been recognized as an effective method for the treatment of intractable wounds. However, its effects on bone healing remain to be elucidated. Our previous study demonstrated that NPWT induced cell proliferation and osteoblastic differentiation of rat periosteum-derived mesenchymal stem cells (P-MSCs). It was reported that following NPWT treatment, the expression of the mechanotransduction molecule integrin $\beta 5$ is increased, indicating that NPWT may serve an active role in fracture healing by enhancing bone formation and reducing bone resorption. The present study sought to further investigate the efficacy of NPWT on the bone regeneration process in a rabbit radial gap-healing model. All rabbits with radial defects were randomly divided into two groups: NPWT and control groups. Continuous negative pressure at $-125 \mathrm{mmHg}$ was applied to all rabbits. Furthermore, X-ray imaging and scoring on day 7, 14, 21 and 28 postoperatively were performed to evaluate new bone formation. Histological changes were determined via hematoxylin and eosin and Masson's trichrome staining at 2- and 4-weeks following surgery. In addition, vimentin-positive cells located in the periosteum were detected via immunohistochemical examination on day 3 post operation. Finally, protein expression levels of vascular endothelial growth factor (VEGF), bone morphogenetic protein (BMP)-2 and osteopontin (OPN) were evaluated
\end{abstract}

Correspondence to: Professor Li Yan, Department of Orthopedics, Wuhan Fourth Hospital, Puai Hospital, Tongji Medical College, Huazhong University of Science and Technology, 473 Hanzheng Street, Wuhan, Hubei 430000, P.R. China

E-mail: swyanli666@hotmail.com

*Contributed equally

Key words: negative pressure wound therapy, bone regeneration, mesenchymal stem cells, vascular endothelial growth factor, bone morphogenetic protein 2 , osteopontin using western blot analysis on the 2 nd and 4th week following NPWT. X-ray and histological examination revealed that the bone-healing processes in the NPWT group were faster compared with the control group. Additionally, compared with the control group, the NPWT group exhibited higher X-ray scores, increased percentage of positive vimentin-stained cells and upregulated expression of VEGF, BMP-2 and OPN proteins. The aforementioned findings suggest that NPWT, under a continuous negative pressure of $-125 \mathrm{mmHg}$, may accelerate bone regeneration by enhancing MSC proliferation, osteoblastic differentiation and VEGF, BMP-2 and OPN expression.

\section{Introduction}

To date, effective treatments, such as negative pressure wound therapy (NPWT), are commonly used to treat various intractable wounds (1-4). NPWT produces a conducive microenvironment for the stimulation of granulation tissue and subsequent wound healing via open-cell foam dressing and negative pressure (5). Several mechanisms of action underlying NPWT have been proposed, including the reduction of wound tissue edema and bacterial colonization, promotion of cell proliferation and increased local blood perfusion (5-8). In addition, NPWT is used as an adjuvant therapy in orthopedic surgery for the treatment of traumatic wounds and surgical incisions (9-12). However, the role of NPWT in bone tissue healing remains to be elucidated.

Bone repair process represents a highly orchestrated series of physiological events, including cellular recruitment, proliferation and differentiation and the involvement of many growth factors (13). Mesenchymal stem cells (MSCs) serve a critical role in fracture healing. Endogenous MSCs are primarily derived from the periosteum, endosteum and marrow cavity (13). Furthermore, MSCs are differentiated into osteoblasts and chondrocytes, which in turn release soluble factors to regulate bone regeneration, ultimately inducing local and distant osteoprogenitor cell activation (13). In addition, mechanical stimulation serves a key role in bone regeneration and remodeling $(14,15)$. A previous study revealed that porous polyurethane foam and suction of NPWT 
induce microdeformations of the wound surface and cellular mechanotransduction. These micromechanical forces may alter the cell shape and increase fibroblast proliferation and differentiation (16). Another study reported that mechanical strain, caused by NPWT, might affect mature dura matter, resulting in activation of bone tissue formation in a rabbit cranial critical-sized defect model. The results revealed that negative pressure-induced mechanical signals (tissue stretching) may promote the differentiation of progenitor cells from the dura to osteoblasts, and then synthesize bone matrix with subsequent mineralization (17). In our previous study, where an in vitro NPWT bioreactor was used, it was demonstrated that short-term NPWT application at $-125 \mathrm{mmHg}$ promoted periosteal-derived mesenchymal stem cell (P-MSCs) and osteogenic differentiation in rats. Additionally, when NPWT is adopted, expression levels of the mechanotransduction molecule integrin $\beta 5$ are increased, suggesting that NPWT promotes bone formation while concurrently reducing bone resorption and inducing fracture healing (18). These findings support the hypothesis that NPWT may result in mechanical strain transduction to the underlying periosteum, mechanical stretching of osteoprogenitor cells and stimulated bone regeneration in traumatic wounds with fractures or segmental bone defects.

In the present study, a rabbit radial gap-healing model was used to investigate the efficacy of NPWT on the bone regeneration process. Following treatment with negative pressure, changes in the expression levels of several critical factors involved in bone formation, such as bone morphogenetic protein (BMP)-2, osteopontin (OPN) and vascular endothelial growth factor (VEGF) were measured. The findings of the present study may provide additional insights regarding the potential mechanism of NPWT action in bone healing.

\section{Materials and methods}

Establishment of an animal model. The present study was approved by the Institutional Animal Care and Use Committee of Huazhong University of Science and Technology. A total of 2 New Zealand rabbits (age, 21-23 weeks; body weight, 3.0-3.5 kg; sex, 18 males and 10 females) were purchased from the experimental animal center of Huazhong University of Science and Technology (Wuhan, China). The rabbits were housed in a light ( $12 \mathrm{~h}$ light/dark cycle), temperature $\left(16^{\circ} \mathrm{C}\right)$ and relative humidity $(40 \%)$ controlled room with free access to food and water for at least 1 week prior to any procedures. All rabbits exhibited mature bone tissue and were intramuscularly anesthetized using $5 \mathrm{mg} / \mathrm{kg}$ xylazine and $35 \mathrm{mg} / \mathrm{kg}$ ketamine (19). The skin preparation and disinfection of the left forelimb were performed according to standard protocols (20). In brief, a 2-3 cm vertical skin incision was performed on the lateral side of the limb at approximately the same distance from the elbow and carpal joint. Subsequently, the surrounding muscles were detached with artery forceps and the radial bone was exposed. A 6-mm long bone section of the central diaphysis of the radius was cut using an electric saw and removed to create a segmental defect (Fig. 1). In addition, the osteotomy site was thoroughly rinsed with saline to remove residual tissue, including fascia, bone and muscle, in the gap. The soft tissue was then reapproximated and the skin was not closed. All wounds were randomly divided into two groups. In the first and second group, the wound was covered with vacuum sealing drainage (VSD) foam (Wuhan VSD Medical Science \& Technology Co., Ltd.) and sterile gauze (control group), respectively. A continuous negative pressure of $-125 \mathrm{mmHg}$ was applied using a tubular suction pump and drainage collection system. For VSD-covered wounds, dressings were replaced on days 3, 7 and 14 following surgery and every 3 days for the gauze-covered wounds. Finally, all rabbits were treated with intramuscular injections of 400,000 units of penicillin daily for 3 days to prevent infection and for analgesia.

$X$-ray imaging and scoring. Mediolateral radiography of the affected sites was performed immediately after surgery and subsequently on days 7, 14, 21 and 28. Each radiography was performed under the same conditions $(1.2 \mathrm{sec}, 50 \mathrm{kV}, 5.4 \mathrm{~mA}$, $50 \mathrm{~cm}$ film-focus distance) using a standard protocol (20). The Lane-Sandhu X-ray scoring system (19) was used for quantitative analysis of new bone formation, extent and size of the callus, bridging of the gap and remodeling signs $(n=5)$.

Histological examination. The bones were collected on the 2nd and 4th week following surgery. The rabbits were sacrificed via air embolism, during which all rabbits were anesthetized by xylazine and ketamine as described earlier. At $10 \mathrm{~min}$ after anesthesia, $10 \mathrm{ml} / \mathrm{kg}$ air was injected into the ear vein. Animal death was confirmed by checking respiration and palpebral, pedal and postural reflexes. A $2.5-\mathrm{cm}$ long section of the radius, including the sites with bone defect and normal bone tissue on both sides, was removed. Subsequently, saline and $10 \%$ neutral formalin solutions were used to rinse and fix samples at $4^{\circ} \mathrm{C}$ for $24 \mathrm{~h}$, respectively. The samples were incubated at $4^{\circ} \mathrm{C}$ for one week and decalcified using $20 \%$ EDTA. The samples were then dehydrated using a gradient of ethanol solutions. Following paraffin embedding, the samples were longitudinally cut into $5 \mu \mathrm{m}$-thick sections and were subsequently stained with hematoxylin and eosin (H\&E) and Masson's trichrome staining at room temperature for $5 \mathrm{~min}$. Finally, the stained sections were observed using an optical microscope (magnification, x100).

Immunohistochemical examination. The periosteum adjacent to the radial gap was collected on day 3 post-surgery. The specimens were fixed, embedded and sectioned using conventional methods (18). Subsequently, specimens were permeabilized with $0.5 \%$ Triton $\mathrm{X}-100$ for $10 \mathrm{~min}$ at room temperature and non-specific epitopes were blocked using $10 \%$ goat serum for $1 \mathrm{~h}$ at $4^{\circ} \mathrm{C}$. Finally, the samples were first incubated with a specific rabbit anti-vimentin antibody (1:100; Wuhan Boster Biological Technology, Ltd. cat. no. M00235-1) overnight at $4^{\circ} \mathrm{C}$ and subsequently with a goat anti-rabbit secondary antibody labeled with streptavidin-biotin complex (1:200; Wuhan Boster Biological Technology, Ltd.; cat. no. BA1034) for $30 \mathrm{~min}$ at $37^{\circ} \mathrm{C}$.

Western blot analysis. Western blot analysis was performed as previously described (18). The bone tissues were collected from the radial gaps on the 2 nd and 4 th week post-surgery. Following freezing of the tissue samples in 

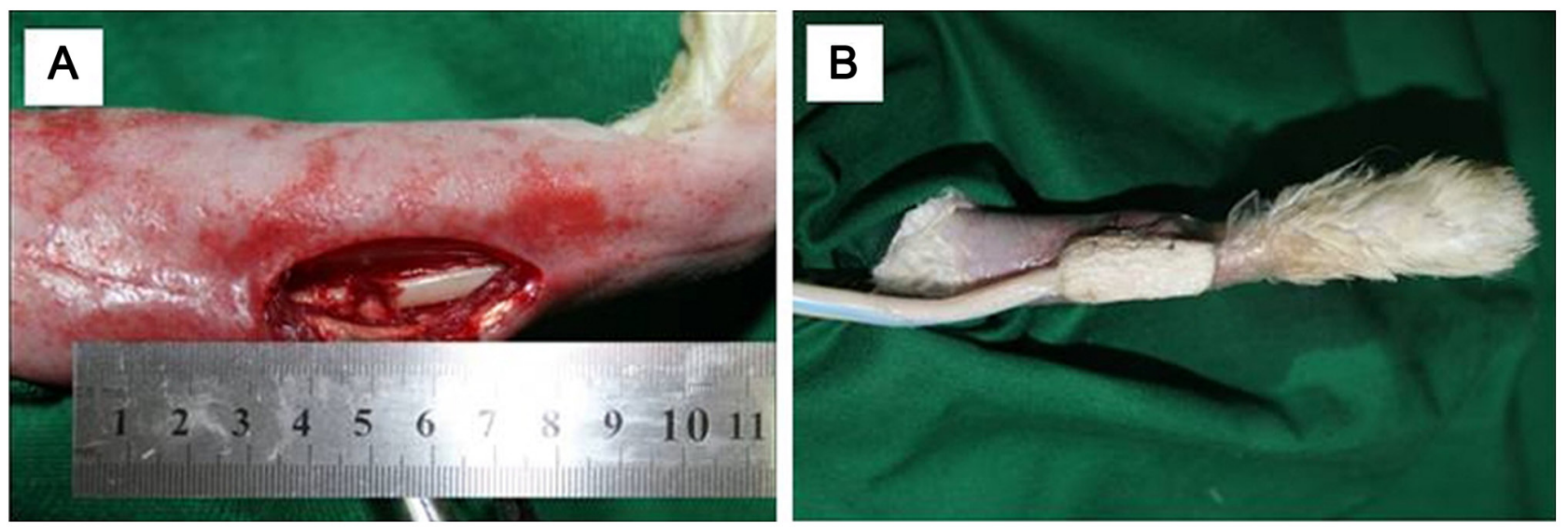

Figure 1. Preparation of the rabbit radial segmental bone defect model. (A) A 6-mm bone defect was created in the central diaphysis of radius. (B) The wounds in the negative pressure wound therapy group were covered with vacuum sealing drainage dressing. Negative pressure was applied continuously at $-125 \mathrm{mmHg}$.

liquid nitrogen, samples were then ground into powder using a pestle and mortar. The cells were lysed using RIPA buffer (Beyotime Institute of Biotechnology) supplemented with phosphatase-inhibitor cocktail (Sigma-Aldrich; Merck KGaA; cat. no. 11873580001) and $1 \mathrm{mM}$ phenylmethylsulfonyl fluoride (PMSF; both Sigma-Aldrich; Merck KGaA). The protein extracts were separated via 6\% SDS-PAGE and subsequently electrotransferred onto PVDF membranes (Hybond-P; GE Healthcare). The membranes were then incubated overnight at $4{ }^{\circ} \mathrm{C}$ with TBS-Tween-20 supplemented with one of the following primary antibodies: Mouse anti- $\beta$-actin (1:1,000; Boster Biological Technology; cat. no. P60709), mouse anti-VEGF (1:200; Santa Cruz Biotechnology, Inc. cat. no. sc-7269), mouse anti-BMP-2 (1:400; Santa Cruz Biotechnology, Inc. cat. no. sc-137087) or mouse anti-OPN (1:400; Abcam; cat. no. ab228748). Following incubation with secondary horseradish peroxidase-conjugated goat anti-mouse IgG antibody (1:1,000; Santa Cruz Biotechnology, Inc. cat. no. sc-2005), protein bands were detected using the enhanced chemiluminescence method. The results were quantified using ImageJ software (version no. 1.48 National Institutes of Health).

Statistical analysis. The results are presented as the mean \pm SD . Statistically significant differences were determined using two-way ANOVA or mixed ANOVA followed by Bonferroni's post hoc test. All statistical analyses were performed using GraphPad Prism 6 (GraphPad Software, Inc.). P $<0.05$ was considered to indicate a statistically significant difference.

\section{Results}

$X$-ray examination. The fracture gap-healing processes was monitored in all rabbits using radiographic examination on days $0,7,14,21$ and 28 (Fig. 2A). The results revealed that calluses were formed around the defects in the NPWT group on day 14 . By day 28, callus formation was increased on the proximal and distal radial defects. The bone defects were radiographically proven to be united. In addition, roentgenographic examination of the control group revealed a small number of peripheral calluses on day 14 and day 21 following surgery. However, on day 28 , the bone callus formation was poor and none of the bone defects completely healed. Subsequent analysis on days 14, 21 and 28 after surgery using the Lane-Sandhu X-ray scoring system demonstrated a significantly higher score in the NPWT group compared with the control group (Fig. 2B). The results indicated that compared with the control group, the osseointegration rate of the NPWT group was significantly increased.

Histological examination. H\&E histological staining was performed at 2 and 4 weeks following surgery. Histopathological examination revealed that in the control group, the majority of bone defects were filled with fibrous tissue, while in the NPWT group, new bone and cartilage island formation was observed (Fig. 3). At the 4th week, small and sparse trabecular bones, mixed cartilage islands and new bone tissue formation were detected in the control group. However, in the NPWT group, the trabecular bones were thicker and denser, with some trabecular bone transforming into mature lamellar bone tissue (Fig. 3). Furthermore, Masson's trichrome staining revealed that connective tissue proliferation occurred at the 2 nd week post-operation in the control group (Fig. 4A), while lots of cartilage islands emerged in the NPWT group (Fig. 4B). By the 4th week, increased mature bone formation (stained red) was observed in the NPWT group (Fig. 4D) compared with the control group (Fig. 4C). All these outcomes were consistent with H\&E results.

Immunohistochemical examination. Histological sections of the periosteum adjacent to the defected sites were collected following surgery and subsequently incubated with antibodies specific against vimentin. On day 3 after surgery, the number of vimentin-positive cells (stained brown) was higher in the NPWT group (Fig. 5A) compared with the control group (Fig. 5B).

$V E G F, B M P-2$ and $O P N$ expression. Western blot analysis revealed that the protein expression levels of OPN, VEGF and BMP-2 significantly increased in the NPWT group compared with the control group at weeks 2 and 4 post operation (Fig. 6). At week 2 following surgery, the expression levels of the aforementioned proteins in the NPWT group were 2-fold higher compared with the control group. Furthermore, at week 

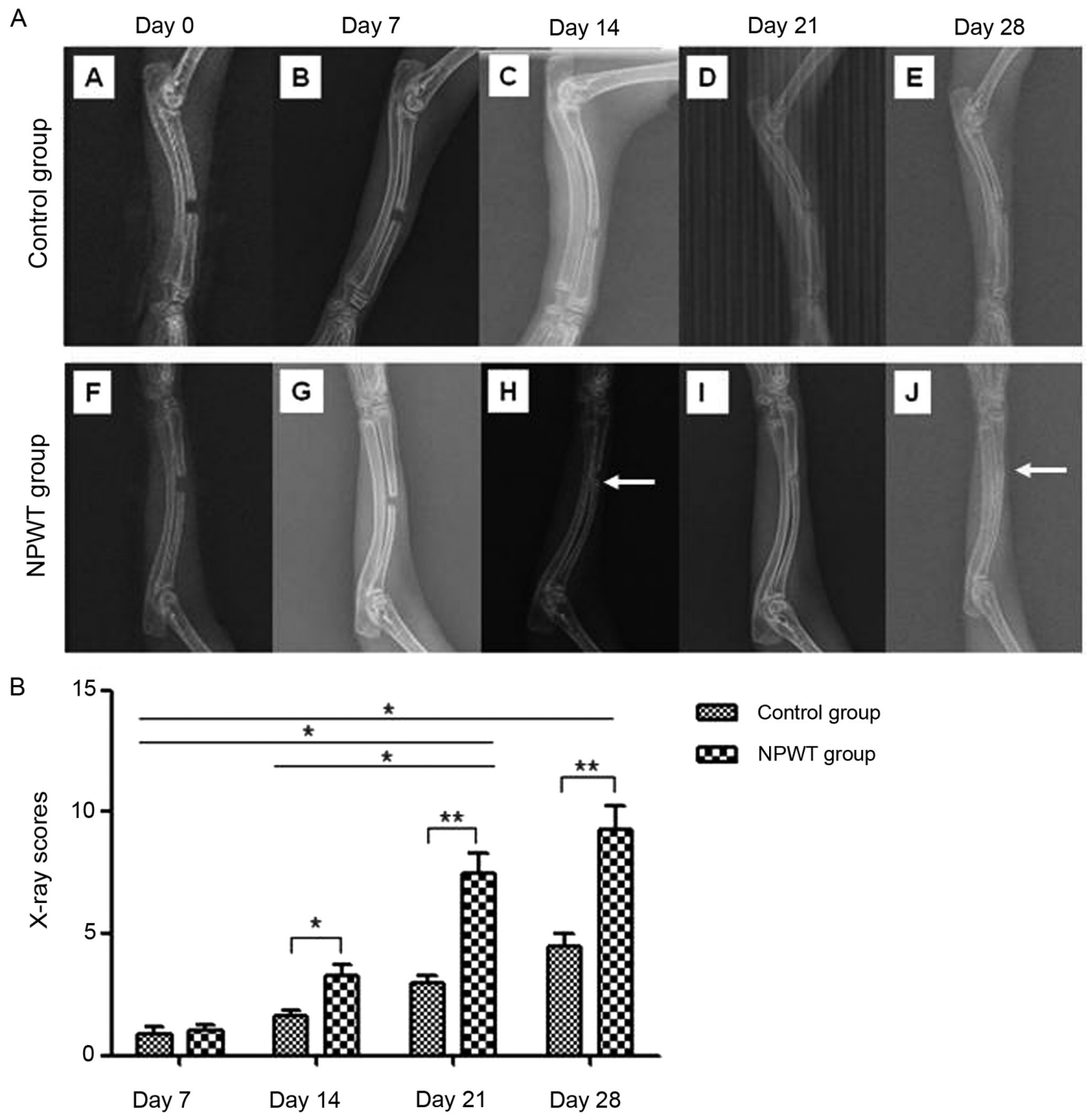

Figure 2. X-ray imaging and Lane-Sandhu scoring. (A) Mediolateral radiographs of the animals in the NPWT group and control group on 0, 7, 14, 21 and 28 days post-operation. (B) Comparison of X-ray scores using the Lane and Sandhu scoring system. Data are presented as the mean \pm standard deviation. $\mathrm{n}=5$. ${ }^{*} \mathrm{P}<0.05$ and ${ }^{* *} \mathrm{P}<0.01$. NPWT, negative pressure wound therapy; VSD, vacuum sealing drainage.

4 post-surgery, the relative expression levels of OPN, VEGF and BMP-2 in the NPWT group were also significantly higher than the control group.

\section{Discussion}

The NPWT approach was first applied in orthopedic surgery in 1993 by Fleischmann et al (21) and was originally used to improve gradual treatment of open fractures associated with soft-tissue defects. More specifically, NPWT has been used to treat Gustilo type III open fractures to achieve primary closure of wounds, which is generally unattainable $(22,23)$. Currently, NPWT has been considered an important adjunct for the treatment of traumatic wounds and surgical incisions associated with orthopedic trauma (9-12). It was reported that NPWT provided wound coverage and maintained incision edges, thus eliminating edema, removing cytotoxic factors and promoting granulation tissue formation $(2,24,25)$. However, its precise mechanism of action needs to be further investigated.
Although the advantages of NPWT have been reported, controversy still exists. A number of systematic reviews have reported that NPWT significantly decreases the risk of infection and accelerates the wound healing process $(26,27)$. However, other studies suggested that negative pressure dressings do not confer any advantage over conventional wound dressings for open fracture treatment (28-30). In addition, several factors may influence the complication rate of NPWT, such as the degree of soft and bone tissue injury, adequate debridement, nutritional support, patients' baseline health status and antibiotic therapy (27).

To date, few studies have investigated the effects of NPWT on bone tissue. Zhang et al (31) demonstrated that intermittent negative pressure promoted bone regeneration in a rabbit skull defect model. Our previous study demonstrated that NPWT induced P-MSC proliferation and osteogenic differentiation (18). In the present study, a rabbit radial gap-healing model was employed to further investigate the role of NPWT in bone tissue healing. Bone regeneration was detected following 


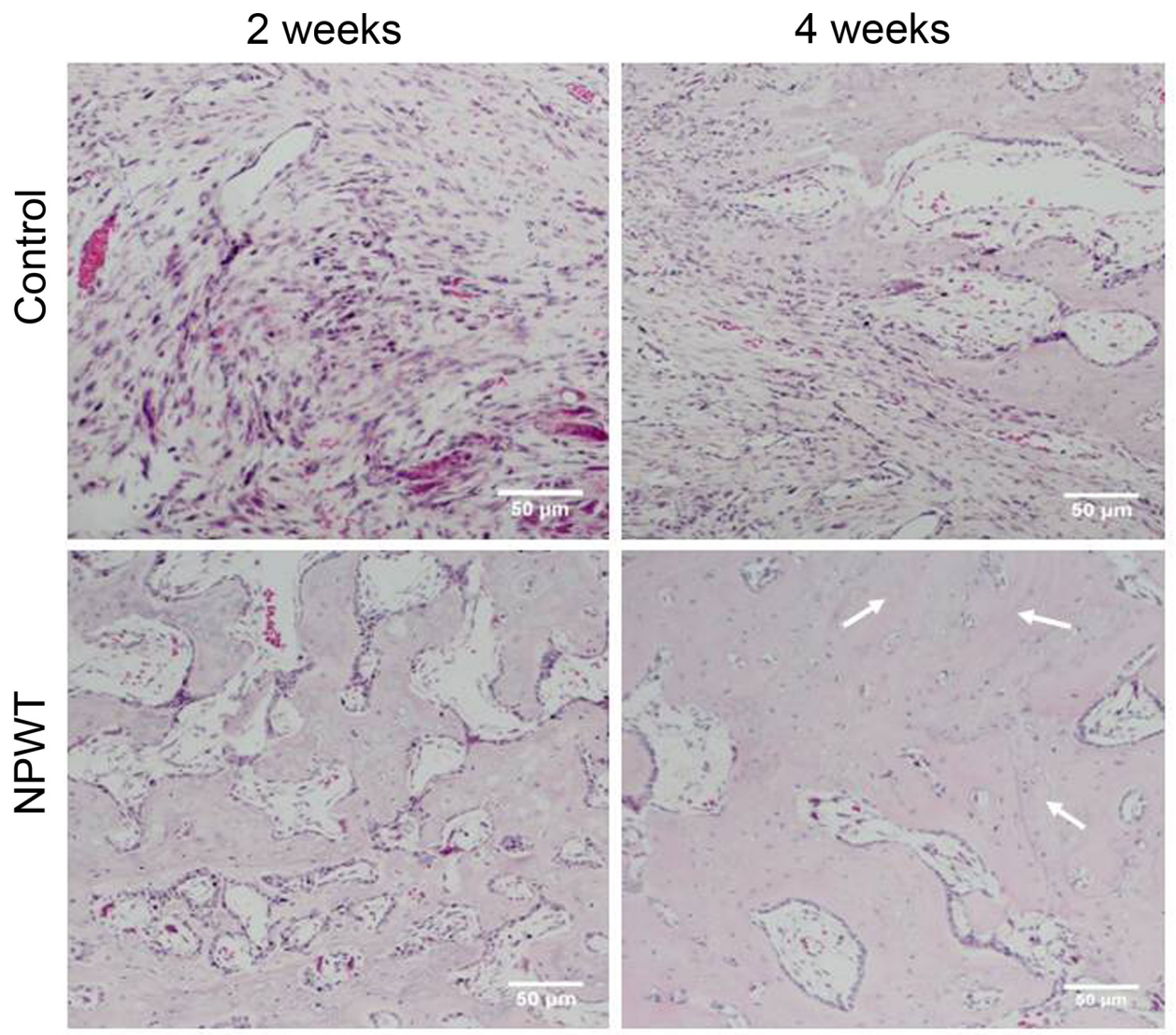

Figure 3. Hematoxylin and eosin staining for histological evaluation of bone regeneration. At 2 weeks post operation, the bone defects were filled with fibrous tissues in the control group, while cartilage islands and new bone were observed in the NPWT group. At 4 weeks post operation, the proportion of trabecular bone in the control group was small, sparse and mixed with cartilage islands and new bone tissues. In the NPWT group, the trabecular bone became thick and dense. Some had become lamellar bone (white arrows). Scale bar, $50 \mu \mathrm{m}$. NPWT, negative pressure wound therapy.
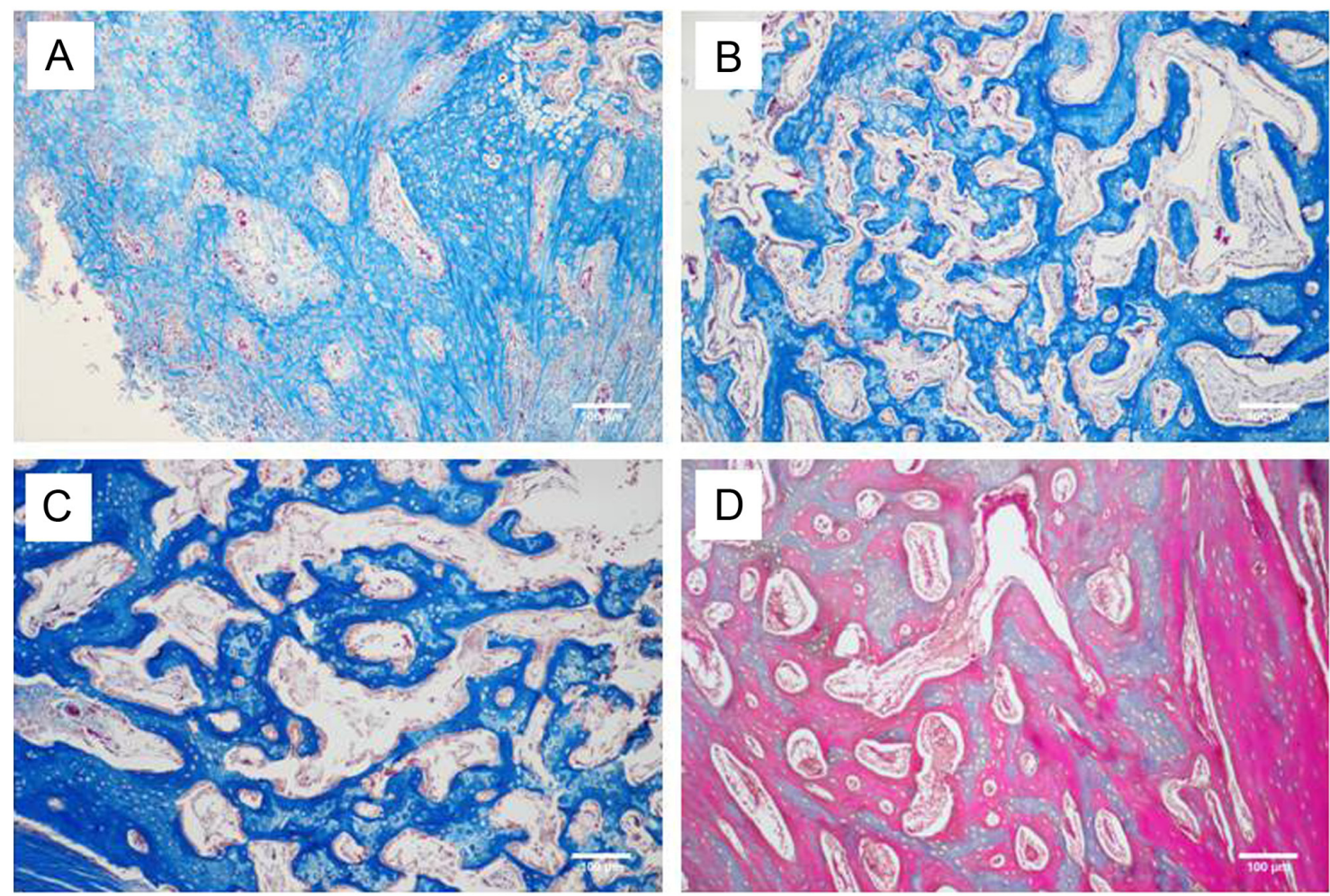

Figure 4. Masson's trichrome staining for histological evaluation of bone regeneration. At 2 weeks post operation, the bone gaps were filled with fibrous connective tissue in the (A) control group, while numerous cartilage islands emerged in the NPWT group (B). At 4 weeks, cartilage islands were found in the control group (C), while mature bone emerged in the NPWT group (D). Scale bar, $100 \mu \mathrm{m}$. NPWT, negative pressure wound therapy. 

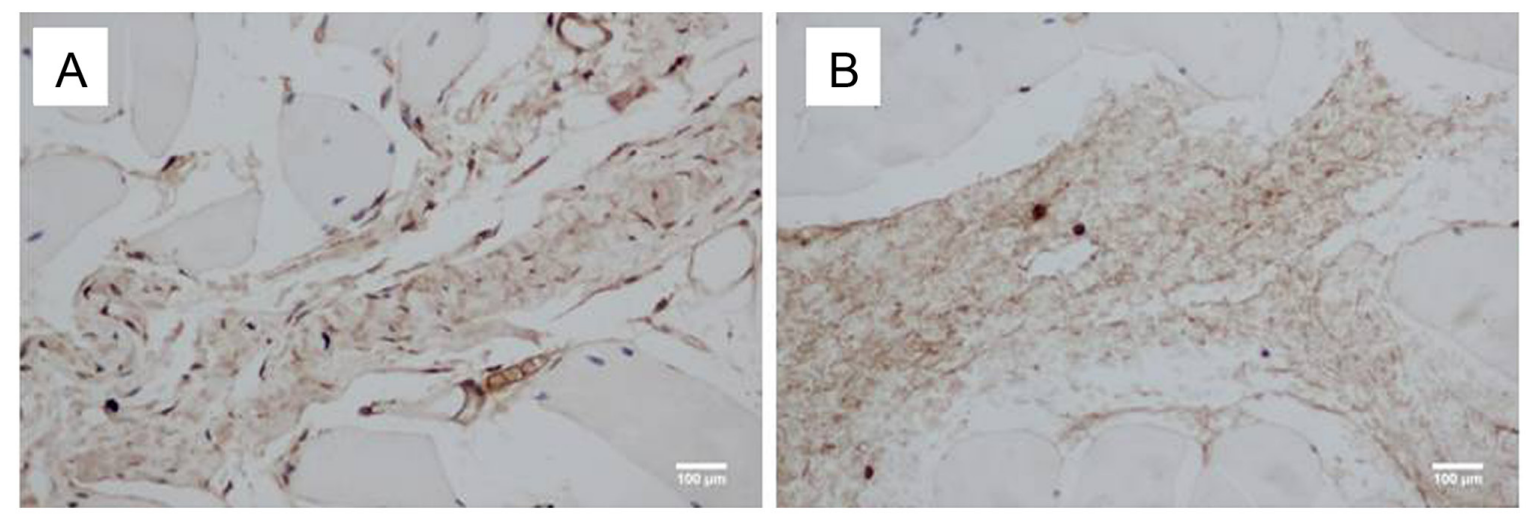

Figure 5. Immunohistochemical observation for MSCs in periosteum. Vimentin-positive cells in (A) the negative pressure wound therapy group and (B) the control group 3 days post-operation. Scale bar, $100 \mu \mathrm{m}$.

A

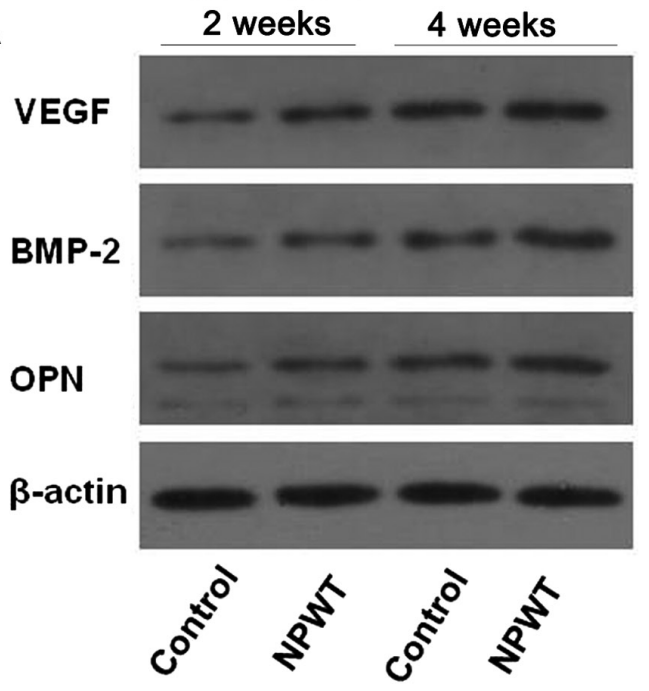

C

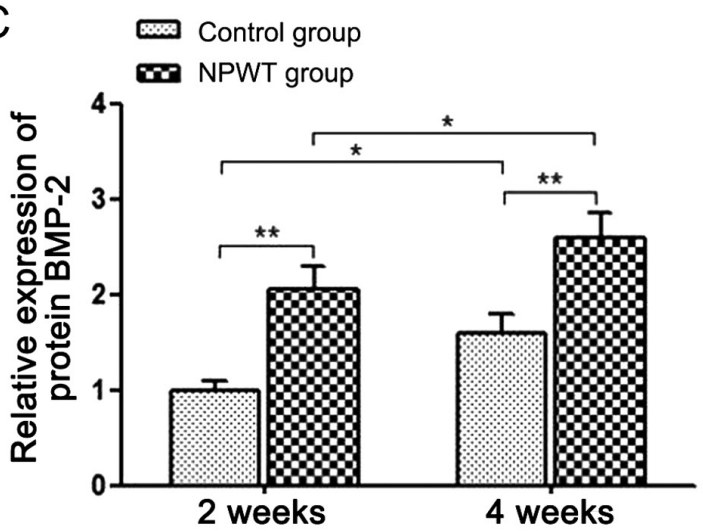

B

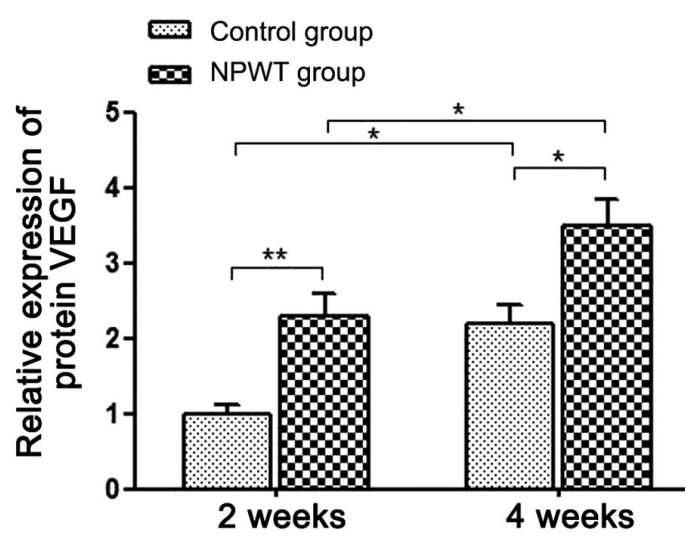

D

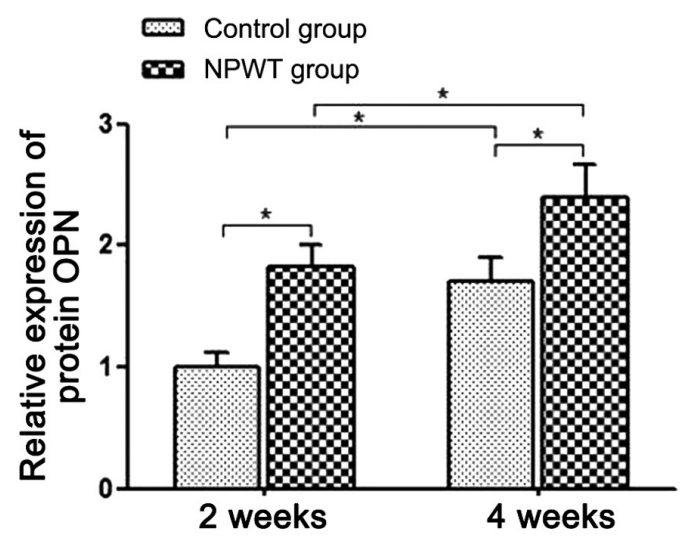

Figure 6. VEGF, BMP-2 and OPN protein expression. (A) Representative western blot image and quantification of (B) VEGF, (C) BMP-2 and (D) OPN protein expression at each time point examined in the NPWT and control groups. "P $<0.05$ and ${ }^{* *} \mathrm{P}<0.01$. VEGF, vascular endothelial growth factor; BMP-2, bone morphogenetic protein 2; OPN, osteopontin; NPWT, negative pressure wound therapy; 2W, week 2; 4W, week 4.

surgery using X-ray and histological staining. The results showed that NPWT, with a continuous suction at $-125 \mathrm{mmHg}$, significantly accelerated bone regeneration compared with the conventional gauze approach. In addition, on the 3rd day following surgery, immunohistochemical staining revealed that the number of vimentin-positive cells was higher in the
NPWT group compared with the control group. Vimentin is an intermediate filament protein that is typically expressed in MSCs and is considered a main marker of rabbit MSCs (32). These findings indicated that NPWT may enhance MSC proliferation in the periosteum, which is consistent with our previous in vitro study (18). 
Western blot analysis results revealed that VEGF protein expression was significantly increased during the bone healing process following negative pressure application. VEGF-induced angiogenesis serves a critical role in bone regeneration and fracture repair. The new capillaries provide the cells in the fracture site with the necessary nutrients to mediate the healing process (33). In addition, it was previously demonstrated that VEGF serves a key role in the recruitment and differentiation of osteoclasts and osteoblasts, respectively (33). The present study also revealed that NPWT upregulated BMP-2 expression during the bone healing process (on the 3rd-4th week post-surgery). BMPs belong to the transforming growth factor- $\beta$ superfamily. Among the members of the superfamily, BMP-7, BMP-4 and BMP-2 are the best-characterized BMPs with osteoinductive properties (34). The aforementioned proteins influence the induction of osteoblasts and chondrocytes differentiation, resulting in membrane enhancement and cartilage ossification, which in turn accelerate bone formation (34). OPN, a key non-collagenous protein, is involved in bone remodeling and mediates the binding of osteoclasts to the bone surface (35). In addition, OPN serves several important roles in the biomineralization process, including modulation of osteoclastic function, osseous cell adherence and matrix mineralization (35). The results of the present study indicate that protein expression levels were increased in the NPWT group compared with the control group at three weeks after surgery. This finding indicated that NPWT-mediated OPN upregulation may influence bone remodeling. Furthermore, OPN and BMP-2 are considered as markers of different stages of MSC differentiation into osteoblasts $(36,37)$. Therefore, NPWT may induce bone formation and osteoblastic differentiation of MSCs via upregulating VEGF, BMP-2 and OPN expression in a rabbit bone healing model.

In the present study, the rabbit segmental bone defect model was directly established using a sharp saw. In patients with open fracture, a high-energy trauma is often associated with severe soft tissue injury, extensive contamination and decreased viability (38). Therefore, further studies on NPWT-mediated bone formation in an open fracture model under high-energy trauma should be performed to verify its osteoinductive effects. Although NPWT exhibits beneficial effects on bone tissue regeneration in rabbits, further research on its mechanisms of action is required. It is also essential to test other pressure values and effect time of NPWT to find optimal conditions for promoting osteogenesis.

In conclusion, the present study revealed that NPWT, with a continuous $-125 \mathrm{mmHg}$ suction, accelerated bone regeneration by upregulating VEGF, BMP-2 and OPN expression levels, and promoting osteoblast differentiation and MSC proliferation. NPWT is considered a promising technique that may be advantageous for wound healing treatment. Finally, further basic and clinical studies focusing on optimizing NPWT are required. It is also important to try other negative pressure waveforms and find the most beneficial conditions for osteogenesis. The current study may help to confirm the effectiveness and elucidate the clinical indications for NPWT in open fracture treatment.

\section{Acknowledgements}

Not applicable.

\section{Funding}

The present study was supported by the Natural Science Fund of Hubei Provincial Health Commission (grant no. 2020CFB464) and Wuhan Municipal Health Commission (grant no. WX20Q15).

\section{Availability of data and materials}

The datasets used and/or analyzed during the current study are available from the corresponding author on reasonable request.

\section{Authors' contributions}

Conceived and designed the experiments: JZ, LY. Performed the experiments: JZ, FW, MW and YA. Analyzed the data: JZ, FW, JW and RH. Contributed to the writing of the manuscript: JZ and FW. JZ and FW confirm the authenticity of all the raw data. All authors read and approved the final manuscript.

\section{Ethics approval and consent to participate}

All experiments were approved by the Institutional Animal Care and Use Committee of Huazhong University of Science and Technology, and the animal procedures were performed in strict accordance with institutional and national guidelines. All efforts were made to minimize suffering.

\section{Patient consent for publication}

Not applicable.

\section{Competing interests}

The authors declare that they have no competing interests.

\section{References}

1. Bee TK, Croce MA, Magnotti LJ, Zarzaur BL, Maish GO III, Minard G, Schroeppel TJ and Fabian TC: Temporary abdominal closure techniques: A prospective randomized trial comparing polyglactin 910 mesh and vacuum-assisted closure. J Trauma 65: 337-344, 2008.

2. Morykwas MJ, Argenta LC, Shelton-Brown EI and McGuirt W: Vacuum-assisted closure: A new method for wound control and treatment: Animal studies and basic foundation. Ann Plastic Surg 38: 553-562, 1997.

3. Armstrong DG and Lavery LA; Diabetic Foot Study Consortium: Negative pressure wound therapy after partial diabetic foot amputation: A multicentre, randomised controlled trial. Lancet 366: 1704-1710, 2005.

4. Fleck T, Gustafsson R, Harding K, Ingemansson R, Lirtzman MD, Meites HL, Moidl R, Price P, Ritchie A, Salazar J, et al: The management of deep sternal wound infections using vacuum assisted closure (V.A.C.) therapy. Int Wound J 3: 273-280, 2006.

5. Argenta LC and Morykwas MJ: Vacuum-assisted closure: A new method for wound control and treatment: Clinical experience. Ann Plastic Surg 38: 563-577, 1997.

6. Javed AA, Teinor J, Wright M, Ding D, Burkhart RA, Hundt J, Cameron JL, Makary MA, He J, Eckhauser FE, et al: Negative pressure wound therapy for surgical-site infections: A randomized trial. Ann Surg 269: 1034-1040, 2019.

7. Liu Y, Zhou Q, Wang Y, Liu Z, Dong M, Wang Y, Li X and $\mathrm{Hu} \mathrm{D}$ : Negative pressure wound therapy decreases mortality in a murine model of burn-wound sepsis involving Pseudomonas aeruginosa infection. PLoS One 9: e90494, 2014. 
8. Orgill DP and Bayer LR: Update on negative-pressure wound therapy. Plast Reconstr Surg 127 (Suppl 1): S105-S115, 2011.

9. Iheozor-Ejiofor Z, Newton K, Dumville JC, Costa ML, Norman G and Bruce J: Negative pressure wound therapy for open traumatic wounds. Cochrane Database Syst Rev 7: CD012522, 2018.

10. Agarwal A: Management of closed incisions using negative-pressure wound therapy in orthopedic surgery. Plast Reconstr Surg 143: S21-S26, 2019.

11. A N, Khan WS and J P: The evidence-based principles of negative pressure wound therapy in trauma \& orthopedics. Open Orthop J 8: 168-177, 2014.

12. Stannard JP, Volgas DA, McGwin G III, Stewart RL, Obremskey W, Moore T and Anglen JO: Incisional negative pressure wound therapy after high-risk lower extremity fractures. J Orthop Trauma 26: 37-42, 2012.

13. Wang X, Wang Y, Gou W, Lu Q, Peng J and Lu S: Role of mesenchymal stem cells in bone regeneration and fracture repair: A review. Int Orthop 37: 2491-2498, 2013.

14. Maycas M, Esbrit P and Gortazar AR: Molecular mechanisms in bone mechanotransduction. Histol Histopathol 32: 751-760, 2017.

15. Duncan RL and Turner CH: Mechanotransduction and the functional response of bone to mechanical strain. Calcif Tissue Int 57: 344-358, 1995.

16. Lu F, Ogawa R, Nguyen DT, Chen B, Guo D, Helm DL, Zhan Q, Murphy GF and Orgill DP: Microdeformation of three-dimensional cultured fibroblasts induces gene expression and morphological changes. Ann Plast Surg 66: 296-300, 2011.

17. Swain LD, Cornet DA, Manwaring ME, Collins B, Singh VK, Beniker D and Carnes DL: Negative pressure therapy stimulates healing of critical-size calvarial defects in rabbits. Bonekey Rep 2: 299, 2013.

18. Zhu J, Yu A, Qi B, Li Z and Hu X: Effects of negative pressure wound therapy on mesenchymal stem cells proliferation and osteogenic differentiation in a fibrin matrix. PLoS One 9: e107339, 2014

19. Udehiya RK, Amarpal, Aithal HP, Kinjavdekar P, Pawde AM, Singh R and Taru Sharma G: Comparison of autogenic and allogenic bone marrow derived mesenchymal stem cells for repair of segmental bone defects in rabbits. Res Vet Sci 94: 743-752, 2013.

20. Liu J, Zhou P, Long Y, Huang C and Chen D: Repair of bone defects in rat radii with a composite of allogeneic adipose-derived stem cells and heterogeneous deproteinized bone. Stem Cell Res Ther 9: 79, 2018

21. Fleischmann W, Strecker W, Bombelli M and Kinzl L: Vacuum sealing as treatment of soft tissue damage in open fractures. Der Unfallchirurg 96: 488-492, 1993 (In German).

22. Takeuchi N, Mae T, Hotokezaka S, Sasaki K, Matsushita A, Miake G, Kuchiishi R and Noguchi Y: A Gustilo type IIIB open forearm fracture treated by negative pressure wound therapy and locking compression plates: A case report. Fukuoka Igaku Zasshi 102: 293-297, 2011.

23. Babiak I: Open tibial fractures grade IIIC treated successfully with external fixation, negative-pressure wound therapy and recombinant human bone morphogenetic protein 7 . Int Wound J 11: 476-482, 2014
24. Suzuki T, Minehara A, Matsuura T, Kawamura T and Soma K: Negative-pressure wound therapy over surgically closed wounds in open fractures. J Orthop Surg (Hong Kong) 22: 30-34, 2014.

25. Stannard JP, Singanamala N and Volgas DA: Fix and flap in the era of vacuum suction devices: What do we know in terms of evidence based medicine? Injury 41: 780-786, 2010.

26. Kim JH and Lee DH: Negative pressure wound therapy vs. conventional management in open tibia fractures: Systematic review and meta-analysis. Injury 50: 1764-1772, 2019.

27. Liu X, Zhang H, Cen S and Huang F: Negative pressure wound therapy versus conventional wound dressings in treatment of open fractures: A systematic review and meta-analysis. Int J Surg 53: 72-79, 2018

28. Costa ML, Achten J, Bruce J, Davis S, Hennings S, Willett K, Petrou S, Jeffery S, Griffin D, Parker B, et al: Negative-pressure wound therapy versus standard dressings for adults with an open lower limb fracture: The WOLLF RCT. Health Technol Assess 22: 1-162, 2018.

29. Crist BD, Oladeji LO, Khazzam M, Della Rocca GJ, Murtha YM and Stannard JP: Role of acute negative pressure wound therapy over primarily closed surgical incisions in acetabular fracture ORIF: A prospective randomized trial. Injury 48: 1518-1521, 2017.

30. Cook R, Thomas V and Martin R: Negative pressure dressings are no better than standard dressings for open fractures. BMJ 364: k4411, 2019.

31. Zhang YG, Yang Z, Zhang H, Liu M, Qiu Y and Guo X: Negative pressure technology enhances bone regeneration in rabbit skull defects. BMC Musculoskeletal Disord 14: 76, 2013.

32. Mazurkevych A, Malyuk M, Bezdieniezhnykh $\mathrm{N}$, Starodub L, Kharkevych Y, Jakubczak A and Gryzinska M: Immunophenotypic characteristics and karyotype analysis of bone marrow-derived mesenchymal stem cells of rabbits during in vitro cultivation. Pol J Vet Sci 20: 687-695, 2017.

33. $\mathrm{Hu} \mathrm{K}$ and Olsen BR: Osteoblast-derived VEGF regulates osteoblast differentiation and bone formation during bone repair. J Clin Invest 126: 509-526, 2016.

34. Majidinia M, Sadeghpour A and Yousefi B: The roles of signaling pathways in bone repair and regeneration. J Cell Physiol 233: 2937-2948, 2018

35. Icer MA and Gezmen-Karadag M: The multiple functions and mechanisms of osteopontin. Clin Biochem 59: 17-24, 2018.

36. Tang Z, Wang Z, Qing F, Ni Y, Fan Y, Tan Y and Zhang X: Bone morphogenetic protein Smads signaling in mesenchymal stem cells affected by osteoinductive calcium phosphate ceramics. J Biomed Mater Res A 103: 1001-1010, 2015.

37. Wang $\mathrm{CY}$, Zhao $\mathrm{BH}, \mathrm{Ai} \mathrm{HJ}$ and Wang YW: Comparison of biological characteristics of mesenchymal stem cells grown on two different titanium implant surfaces. Biomed Mater 3: 015004, 2008.

38. Streubel PN, Stinner DJ and Obremskey WT: Use of negative-pressure wound therapy in orthopaedic trauma. J Am Acad Orthop Surg 20: 564-574, 2012.

This work is licensed under a Creative Commons Attribution-NonCommercial-NoDerivatives 4.0 International (CC BY-NC-ND 4.0) License. 